\title{
De MUSE en ARGOLIDE, ou la codicologie à l'ère du numérique
}

\section{Claire Bustarret et Serge Linkès}

\section{OpenEdition}

12 Journals

Édition électronique

URL : http://journals.openedition.org/recherchestravaux/97

DOI : 10.4000/recherchestravaux.97

ISSN : 1969-6434

Éditeur

UGA Éditions/Université Grenoble Alpes

\section{Édition imprimée}

Date de publication : 15 juin 2008

Pagination : 119-136

ISBN : 978-2-84310-125-0

ISSN : 0151-1874

Référence électronique

Claire Bustarret et Serge Linkès, " De MUSE en ARGOLIDE, ou la codicologie à l'ère du numérique », Recherches \& Travaux [En ligne], 72 | 2008, mis en ligne le 15 décembre 2009, consulté le 03 février 2021. URL : http://journals.openedition.org/recherchestravaux/97 ; DOI : https://doi.org/10.4000/ recherchestravaux.97 


\section{De MUSE en ARGOLIDE, ou la codicologie à l'ère du numérique}

L'évolution récente des rapports entre éditeur et manuscrit, à l'ère du numérique, tend à privilégier l'accessibilité et la maniabilité de l'objet virtuel, reléguant l'original hors de portée afin de mieux le préserver'. L'analyse matérielle du manuscrit relève quant à elle d'une démarche descriptive héritée des érudits médiévistes, qui avaient dénommé «codicologie» cette discipline qu'ils ont d'abord appliquée au codex de parchemin ou de papier, et que d'autres chercheurs ont su adapter au manuscrit moderne ${ }^{2}$. Ces deux approches d'un même objet peuvent sembler diamétralement opposées, sur un plan tant pratique que théorique. En effet, à cause des moyens importants qu'elles nécessitent, les campagnes systématiques de numérisation de manuscrits tendent à s'appuyer sur l'argument de la substitution : au lieu de consulter l'original, on consultera désormais sans dommage l'objet virtuel, image «à l'identique» de l'original. La numérisation favorise ainsi la conservation du document. Certes, par rapport aux procédés antérieurs, photocopie, photographie ou microfilm,

I. En complément de cet article, un diaporama a été mis en ligne sur le site de la MSHAlpes : http://www.msh-alpes.prd.fr/Actualites/Manuscrits.htm.

2. À la suite d'une première définition du terme donnée par A. Dain en I 949 dans Les Manuscrits, cette discipline s'est orientée vers une approche archéologique des documents écrits - surtout antiques et médiévaux : voir J.-Ch. Lemaire, Introduction à la codicologie, Louvainla-Neuve, UCL, I989. Pour son application aux manuscrits modernes, voir L. Hay, «Éléments pour l'étude des manuscrits modernes», Codicologica I, I976, p. 91-IO8 et les chapitres «Objets matériels» (M. Bockelkamp) et «Les manuscrits au laboratoire», Les Manuscrits des écrivains, sous la direction de L. Hay, Hachette / CNRS éditions, 1993. 
il s'agit avec le numérique d'un changement radical d'échelle dans la précision et la fiabilité de la reproduction. Mais la plupart des indices matériels permettant d'identifier par exemple un type de papier sont perceptibles au toucher ou grâce à l'éclairage par transparence, et n'apparaissent nullement à l'image. La reproduction comporte donc, pour les codicologues et pour ceux qui souhaitent exploiter le manuscrit non seulement comme objet intellectuel ou esthétique mais aussi comme objet matériel, une perte d'information définitive.

Définitive, mais non irrémédiable. Car si l'on veut compenser une telle perte, les campagnes de numérisation devraient justement s'accompagner de relevés codicologiques aussi complets que possible - sachant qu'il ne s'agit ici que de relevés d'observation, ne comportant aucun prélèvement ni intervention physique ou chimique sur le manuscrit. Ainsi allié à la description matérielle, le fac-similé numérique pourrait mieux prétendre se substituer à l'objet tridimensionnel, et le recours à l'original en serait d'autant moins nécessaire, ce qui conforterait la légitime exigence de préservation des manuscrits. Rappelons que le manuscrit moderne, et particulièrement lorsqu'il s'agit d'un brouillon, est un objet composite, témoin de multiples altérations et remaniements, qui ne se laisse nullement appréhender par une séquence linéaire d'images bidimensionnelles. Fournir au lecteur, en même temps qu'il accède aux reproductions numériques des manuscrits, une vision globale de la composition du support (carnet, cahier ou liasses de feuilles volantes, comportant d'éventuels découpages et collages, etc.), de l'agencement des feuillets les uns par rapport aux autres, des divers niveaux d'intervention du ou des scripteurs, s'avère en ce domaine plus qu'un «enrichissement» de l'information, une forme de structuration indispensable. Intégrer l'apport des indices codicologiques au niveau de l'enregistrement du document sous forme numérique suppose toutefois certaines exigences techniques. C'est le sens de la participation de l'équipe «Techniques et pratiques de l'écrit» au nouveau projet OPTIMA, récemment présenté par l'ITEM au programme thématique «Corpus et outils de la recherche en sciences humaines et sociales» sous l'égide de l'ANR'.

Nous avons pu constater, à partir de l'expérience de la base de données MUSE4, à quel point un outil de travail efficace pour la collecte de données codicologiques sur les corpus de manuscrits littéraires modernes (XVIII $\mathrm{e}^{\mathrm{e}}$ $\mathrm{XX}^{\mathrm{e}}$ siècles) pouvait contribuer aux recherches génétiques et à la préparation

3. OPTIMA, acronyme du programme «Outils Pour le Traitement et l'analyse de l'Information dans les MAnuscrits modernes », répondant à l'appel d'offres de l'Agence nationale de la recherche 2006.

4. MUSE : «Manuscrits, Usages des Supports et de l'Écriture», base de données illustrée de reproductions bétaradiographiques des filigranes, développée par S. Linkès et $\mathrm{Cl}$. Bustarret dans le cadre du programme collectif du CNRS intitulé Archives de la création, 1998-2000. 


\section{DE MUSE EN ARGOLIDE}

d'éditions critiques. La présentation qui suit entend donner un aperçu de son fonctionnement actuel et de ses applications à la recherche, témoignant d'une meilleure exploitation des données qui, dans la genèse littéraire, relèvent du "paradigme indiciaire», selon l'expression de Carlo Ginzburgs.

La constitution de bases de données codicologiques et génétiques couplées à la numérisation et à la transcription des manuscrits doit-elle se contenter, au fur et à mesure que les œuvres sont numérisées, d'enrichir l'édition électronique par rapport à l'édition imprimée? Avec le développement en cours d'un auxiliaire de recherche plus complet, intitulé ARGOLIDE', nous souhaitons fournir un véritable instrument de travail qui puisse contribuer à l'élaboration de bases de connaissances fiables et structurées, accessibles à moyen terme sur Internet.

\section{La base MUSE : un outil codicologique adapté à l'analyse génétique}

Les principales fonctions dévolues à une base de données codicologiques telle que MUSE sont tout d'abord de permettre au chercheur d'effectuer l'analyse matérielle et la description rigoureuse du manuscrit examiné. Sur ce point, nous avons cherché à intégrer dans notre modèle descriptif tous les critères permettant de distinguer les catégories de supports d'écriture en usage pendant les trois derniers siècles ${ }^{7}$, du cahier relié aux feuilles volantes et aux fragments découpés, du carnet de poche au bloc-notes «Sténo» de format $A_{5}$, mais aussi les différents types de papier ${ }^{8}$, du vergé à la forme du XVIII ${ }^{\mathrm{e}}$ siècle aux bifeuillets de vélin quadrillé des cahiers d'écolier industriels du $\mathrm{XX}^{\mathrm{e}}$ siècle et au papier $\mathrm{A}_{4}$ pour machine à écrire. Il est tout aussi important de pouvoir distinguer les mains des scripteurs et les types de tracés, caractérisés par l'emploi d'instruments variés, de la plume au stylo à bille et au feutre9. Recueillir ces données détaillées feuillet par feuillet sur des milliers d'occurrences dépasse de

5. C. Ginzburg, Mythes, emblèmes, traces, Flammarion, I989.

6. ARGOLIDE : «Auxiliaire de Recherche en Génétique des CEuvres Littéraires par l'Interprétation des Données d'Expertise».

7. Voir Cl. Bustarret, "Les manuscrits littéraires modernes et leurs supports», Histoire de l'écriture, de l'idéogramme au multimédia, sous la direction d'A.-M. Christin, Flammarion, 200 I, p. 332-339.

8. Par «type de papier», on entend le modèle de toutes les feuilles de papier produites par la même forme (ou paire de formes quasi identiques) employée dans la fabrication manuelle. Pour la production industrielle, la notion perd de sa pertinence au niveau technique puisque le papier est produit «en continu», les formats étant découpés après séchage : on se réfère alors au produit commercial identifié par format, couleur, texture, grammage et éventuellement filigrane.

9. Voir Cl. Bustarret, «Les instruments d'écriture, de l'indice au symbole», Genesis, ñ ${ }^{\circ}$, printemps I997, p. I75-191. 
loin le simple inventaire fourni par la plupart des catalogues existants. Et la multiplicité des critères empêche le chercheur d'identifier trop rapidement une sorte de papier à la seule présence d'un filigrane ou d'après sa couleur, sans tenir compte de l'épaisseur, du format, ou de la qualité de la pâte.

Dans un second temps, le fonctionnement de la base est conçu pour traiter les données et faciliter les comparaisons entre elles. Les requêtes portant sur les dimensions ou l'épaisseur d'un papier, l'alternance des couleurs d'encre ou de sortes de papier différentes au sein d'un volume, la date de fabrication ou la provenance géographique du support parfois indiquées par le filigrane, sont autant d'éléments qui favorisent des recoupements parfois inattendus et permettent de mieux comprendre le corpus étudié, de formuler des hypothèses ou de remettre en cause des interprétations admises concernant la façon de travailler de tel écrivain ${ }^{10}$.

Enfin, et ce n'est pas la moindre de ses fonctions, on n'attend pas seulement d'une base de données un contrôle de l'enregistrement des données, mais une capacité de stockage quantitativement supérieure à celle de la mémoire du chercheur... C'est donc grâce à la structuration des données et à la stabilité de l'enregistrement qu'une telle base peut prétendre, à mesure qu'elle se constitue, remplir déjà la fonction d'outil de référence fiable. Les relevés codicologiques ne portent pour l'instant que sur deux mille types de papier durant trois siècles d'usage; mais pour chaque volume manuscrit dépouillé, la base MUSE s'avère beaucoup plus complète qu'un répertoire de filigranes ${ }^{11}$, puisqu'elle fournit de nombreuses autres caractéristiques du papier, ainsi qu'un recueil d'occurrences détaillées feuillet par feuillet, et parce qu'elle fournit, en sus de l'identification du papier, plusieurs autres éléments caractérisant le support.

ı. Voir par exemple C. Volpilhac-Auger, avec la collaboration de Cl. Bustarret, L'Atelier de Montesquieu, Manuscrits inédits de La Brède, Naples, Liguori Editore, Oxford, Voltaire Foundation, 200 I et Condorcet, Tableau historique des progrès de l'esprit humain, édition critique, INED, 2004.

I I. Les répertoires sont des ouvrages d'érudition qui comportent de nombreuses reproductions de filigranes datés, assorties d'index des fabricants et de notices historiques et iconographiques. Citons Ch.-M. Briquet, Les Filigranes. Dictionnaire bistorique des marques du papier dès leur apparition vers I282 jusqu'en I600 [éd. originale 1907], éd. fac-similé par A. Stevenson, 4 volumes, Amsterdam, Paper publications society, I 968 ; E. Heawood, Watermarks, mainly of the $17^{\text {th }}$ and $18^{\text {th }}$ centuries, Hilversum, the Paper publications society, I950; W. A. Churchill, Watermarks in Paper in Holland, France, etc. in the XVIT $T^{\text {th }}$ and XVIII centuries and their interconnection, Amsterdam, Menno Hertzberger, I935; A. Nicolaï, Histoire des moulins à papier du Sud-Ouest de la France : 1300-1800 [...], Bordeaux, G. Delmas, I 935 [rééd. Pau, éd. PyréMonde, 2006]. Plus récemment, R. Gaudriault, Filigranes et autres caractéristiques des papiers fabriqués en France aux XVIT et XVIII siècles, CNRS éditions, J. Telford, I995, et P. Delaunay, Catalogue des filigranes relevés sur des papiers d'archives d'Awvergne, Clermont-Ferrand, Académie des sciences, belles-lettres et arts de Clermont-Ferrand, I997. Signalons que la production des XIX ${ }^{\mathrm{e}}$ et XXe siècles n'y est guère abordée. 
Parmi les données codicologiques issues de l'analyse matérielle, certaines fourniront des informations indispensables pour constituer le dossier génétique. Lorsqu'il étudie les aléas de la numérotation des feuillets (paginations et foliotations autographes, allographes et institutionnelles) et souhaite comparer les états successifs rencontrés afin d'établir la genèse du texte, le chercheur peut ainsi étayer sur les données codicologiques (supports et tracés) ses premières hypothèses de classement. La base MUSE lui permet aussi de «tester» ses options de classement en vérifiant leur cohérence du point de vue de l'analyse matérielle. Une fois classés les manuscrits, et établies les phases de la genèse, le spécialiste peut également recourir à une base adéquate pour corroborer les informations et préparer une édition génétique. Sur un corpus ainsi documenté de façon approfondie, la perspective critique se trouve étayée par un faisceau d'informations vérifiables, et ce d'autant plus que le nombre des manuscrits analysés dans la base de travail permet de comparer les processus suivis pour diverses œuvres, et par plusieurs auteurs. En effet, l'avantage d'un tel outil est de favoriser une prise en compte systématique des données factuelles, qui permet un catalogage détaillé des fonds existants.

\section{Description de l'objet manuscrit}

Rappelons que les manuscrits sous la forme actuelle de conservation ne correspondent pas toujours aux supports d'écriture tels qu'ils ont été initialement utilisés, et que leur disposition ne traduit que fort rarement une intention d'auteur. À nous de discerner les effets des interventions ultérieures à la rédaction, et souvent posthumes : au-delà des tris, des annotations, des reliures, des opérations de restauration, il s'agit de retrouver les traces des gestes qui ont accompagné l'utilisation d'un cahier ou d'une liasse de feuilles volantes au cours de la genèse, tels que plier, retourner, découper, arracher, épingler, coller à l'aide de cire ou de ruban adhésif... Autant de critères d'utilisation qui doivent venir compléter la simple identification du type de support (cahier d'écolier, carnet, bloc-notes, feuilles volantes de divers formats et qualités, papier à lettres ou d'emballage, dos d'enveloppes, pages imprimées arrachées d'un livre ou d'un magazine, etc.) effectuée grâce aux critères de fabrication. Afin d'être en mesure de retrouver la provenance d'un feuillet isolé détaché de tel ou tel support, il est nécessaire d'entrer dans les moindres détails de la nature et de la composition de tous les supports employés ${ }^{12}$.

I 2. Le meilleur modèle d'une telle démarche, appliquée à l'œuvre graphique de Turner, nous est offert par le remarquable ouvrage de P. Bower: Turner's later Papers : a Study of the Manufacture, Selection and Use of his Drawing Papers, (2 volumes, I 787 -I 820 , I $820-$ I 85 I), New Castle, Oak Knoll press, London, Tate Gallery, I 990 et 1999 
L'enquête sur l'emploi des instruments d'écriture est moins aisée à effectuer sans le recours aux analyses physiques ou chimiques. La période de fabrication artisanale présente l'inconvénient de tracés peu stables, du fait de la composition des encres et de la taille périodique des plumes. La période de fabrication industrielle pose au contraire le problème d'objets standardisés, interchangeables : deux stylos à bille du même modèle employés par la même personne n'offrent généralement aucun signe distinctif au niveau du tracé... à moins d'un défaut de fonctionnement. Il s'agit donc dans un premier temps de noter les différences pertinentes à l'intérieur d'un corpus donné (plume/ bille/feutre/crayon à papier; couleurs, épaisseur et souplesse de la pointe), qui souvent suffisent à distinguer différentes interventions d'une même main. En effet, le choix d'instruments différents constitue souvent pour l'écrivain lui-même un moyen pour dissocier les étapes du travail s'inscrivant sur le même support. Cependant, la part d'aléatoire qui entre dans de tels choix est souvent compensée, pour l'observateur attentif, par le recoupement systématique, grâce à la base de travail, entre les données concernant les instruments et l'identification du papier ${ }^{13}$. C'est du moins l'hypothèse qui justifie l'enregistrement descriptif des tracés dans la base en l'état actuel, même de façon sommaire, sous réserve de développements ultérieurs envisageables sur certains corpus. Les manuscrits qui comportent des difficultés d'attribution à différents scripteurs (copistes, secrétaires) bénéficieront assurément du recours aux reproductions numérisées, grâce aux fonctions d'agrandissement et de comparaison d'images côte à côte. L'image ici est indispensable à l'analyse critique. Les progrès déjà enregistrés dans l'identification des «mains» par le recours aux données codicologiques enregistrées dans la base MUSE, s'agissant par exemple des nombreux secrétaires de Montesquieu, pourront ainsi évoluer vers un plus grand degré de certitude.

Quant à l'analyse des papiers, rappelons que notre formulaire descriptif, déjà publié ailleurs ${ }^{14}$, comporte deux séries de critères bien distincts. D’une part les critères de fabrication, qui permettent l'identification de différents types de produits papetiers vendus dans le commerce : chaque type identifié permet de rassembler virtuellement un plus ou moins grand nombre d'occurrences qui peuvent être aujourd'hui éparses dans le corpus conservé. S'agit-il de

I 3. Voir le cas de la campagne d'écriture à l'encre verte étudiée par N. Cavaillès dans «Le Corrupteur corrompu, Barbarie et méthode dans l'écriture cioranienne», Genesis, n' 25, 2005, p. 8 5-106.

I4. Voir notamment $\mathrm{Cl}$. Bustarret, " Mobilité des supports, dynamique de l'écriture : l'apport des indices matériels», Montesquieu, Emvre ouverte? I748-1755 : actes du colloque de Bordeaux, C. Larrère éd., (Cahiers Montesquieu, 9), Naples, Liguori editore; Oxford, Voltaire Foundation, 2005, p. 229-252. 
papiers vergés ou vélins, de fabrication manuelle ou mécanique, de supports destinés ou non à l'écriture, présentant une surface vierge ou imprimée (réglure, quadrillage, en-tête, ou autres caractères imprimés)? Et d'autre part les critères d'utilisation, qui concernent l'emploi singulier de ces matériaux par tel ou tel scripteur, dans le contexte d'un dossier donné - on s'intéresse alors au niveau de chaque occurrence d'un type, donc de chaque feuillet décrit dans le détail. Tels feuillets initialement solidaires à la couture d'un cahier (occurrences désignées comme «bifeuillets») ont-ils été séparés? Tel fragment correspond-il au quart ou au tiers du format initial, a-t-il été découpé avant ou après l'inscription?

Dans la première série de données, le protocole descriptif comporte une dizaine de critères observables sans instrumentation, comme la qualité, la couleur, l'opacité, l'épair ${ }^{15}$, l'éventuelle présence de filigrane, de timbre sec, et d'éléments imprimés (couleur de la réglure ou quadrillage, filet de marge). En revanche des critères tels que la composition (physique et chimique) de la pâte ne sont guère accessibles sans recours au microscope. Aussi les critères mesurables, un peu moins nombreux, sont-ils restreints : les dimensions du feuillet ou de la feuille entière, l'écart entre lignes de chaînettes pour les vergés, nombre de lignes de la réglure ou du quadrillage, dimensions des marges; il est également utile de mesurer l'épaisseur (à l'aide d'un micromètre) et si possible la rugosité (grâce au perthomètre), alors que l'évaluation de l'épair reste effectuée à l'œil nu, donc sujette à l'approximation, faute de recourir à un instrument spécifique, encore peu accessible à ce jour.

Les caractéristiques d'utilisation regroupent tout ce qui concerne les modalités d'assemblage (une liasse peut être simplement pliée, ou bien agrafée, cousue, épinglée, retenue par un trombone...), de pliage (diverses occurrences d'un même type peuvent se présenter tantôt in-folio, tantôt in- $4^{\circ}$ ou in- $8^{\circ}$ ), de découpage et de collage. Ici encore, une description aussi détaillée peut sembler laborieuse en regard d'une image numérisée que l'on croira «explicite». Pourtant seul le passage par l'expression verbale garantit la possibilité de comparer, grâce à l'outil informatique, les différents cas de figure répertoriés afin d'élaborer une synthèse. De plus, l'expérience de ces relevés prouve que seule la contrainte d'un protocole descriptif exigeant garantit une observation complète. Enfin, diverses formes de marquage font l'objet d'un relevé, qu'il s'agisse de la numérotation des pièces (foliotées et/ou paginées par l'écrivain et/ou par des tiers, dont l'institution de conservation), ainsi que plusieurs aspects caractéristiques de la mise en page (modalités d'inscription au recto seul ou rectoverso, respect ou non des lignes et marges imprimées, etc.).

I 5 . Épair : aspect de la structure du papier observable par transparence. 


\section{Le système d'information de la base MUSE}

Si le chercheur littéraire souhaite concevoir une base de données, il doit d'abord accepter d'adopter les stratégies de développement des outils qui sont en vigueur dans l'univers informatique. Ainsi, tout informaticien sait qu'il est impossible d'élaborer une base de données sans passer par l'étape préalable de la conception du système d'information ${ }^{16}$. Ce système permet non seulement d'organiser les données (organisation des données au sein des fichiers et des relations entre les fichiers), mais aussi d'intégrer les contraintes externes à l'outil comme ses conditions d'utilisation, les utilisateurs en amont et en aval, les protocoles de saisie des données, les opérations de maintenance et de sauvegarde. Ce système doit donc être élaboré en étroite collaboration avec tous les utilisateurs afin de tenir compte des besoins, des attentes et des contraintes de chacun. Dans le cas de MUSE, ce travail a été réalisé en présence d'utilisateurs potentiels étudiant des corpus de nature et d'époques différentes. L'organisation des données dans chaque fichier ainsi que les termes descriptifs et critiques à utiliser ont été adaptés à cette diversité qui est inhérente à l'objet d'étude. Une attention particulière a été portée à la définition des relations entre les fichiers qui est garante de l'intégrité de la base ${ }^{17}$.

L'autre aspect de la mise en place du système d'information concerne le choix du logiciel permettant d'élaborer une base de données. Ce choix représente un enjeu considérable, c'est même l'un des éléments essentiels du cahier des charges puisqu'il conditionnera la compatibilité, la pérennité, et l'évolution de la base de données. N'oublions pas que le but d'une base est aussi de communiquer des données même si l'interface peut paraitre moins conviviale que certaines interfaces Web (PHP, XML...) très communes aujourd'hui ${ }^{18}$. Notre choix a été conditionné, à l'époque de la conception, par un souci de compatibilité entre platesformes PC et Macintosh qui nous a orientés vers FileMaker ${ }^{\circledR}$ Pro $^{19}$.

Même si MUSE sert avant tout à stocker les données collectées lors de la description, précédemment évoquée, de l'objet manuscrit, cette base est bien plus qu'une simple banque de données d'information codicologique. Il semble

I6. Pour plus de précisions sur les aspects techniques de la base MUSE, voir Cl. Bustarret et S. Linkès, «Un nouvel instrument de travail pour l'analyse des manuscrits : la base de données MUSE», Genesis, n² 2 , 2003, p. I6I-177.

17. Voir ci-dessous «Structure de la base et intégrité référentielle».

I 8. La base MUSE a été conçue à partir de 1996.

I 9. Il faut également signaler que ce logiciel était accompagné d'un module (Runtime) permettant de créer des applications autonomes libres de droit à partir de la base de données réalisée, ce qui nous a permis de multiplier les versions de MUSE sur différents postes de travail. 
nécessaire de le préciser, car ce n'est pas sans incidence sur la conception même de l'application; c'est aussi ce qui la distingue nettement de la plupart des bases développées en recherche littéraire, qui sont avant tout des bases informatives proposant une interface de consultation donnant accès à des données d'ores et déjà validées. MUSE a suivi un principe différent, puisque c'est l'une des rares applications qui a pour but de construire son objet d'étude : le manuscrit littéraire.

Si construire un objet d'étude littéraire à partir d'une application informatique peut paraittre paradoxal au premier abord, il faut considérer, pour saisir la démarche qui est la nôtre, que MUSE a été avant tout conçue comme un système qui gère l'analyse des données descriptives afin de permettre la formulation d'hypothèses génétiques (fig. I).

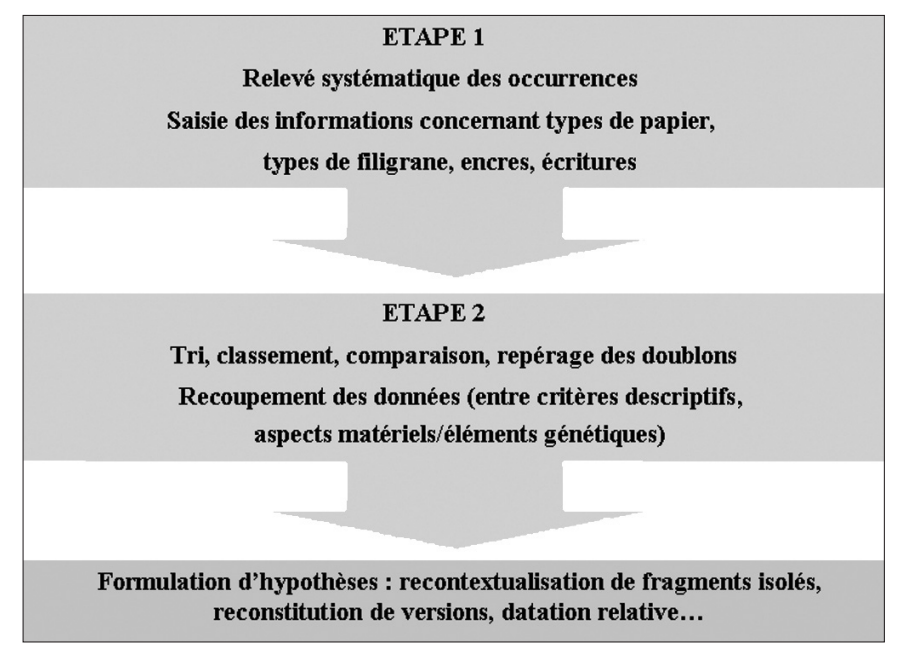

Fig. I. Démarche méthodologique de MUSE.

En procédant par étapes, l'emploi de MUSE doit permettre de proposer des hypothèses utiles à la constitution du dossier de genèse à partir de la multitude des données codicologiques collectées lors de l'analyse matérielle. C'est à partir du croisement des informations concernant chaque feuillet que pourront être identifiés les différents types de papier et les différentes interventions scripturales. La répartition des occurrences entre les différents types de papier et le repérage des interactions avec les opérations d'écriture interviennent donc au terme de la description codicologique exhaustive d'un ou plusieurs volumes manuscrits, complétée le cas échéant par l'apport des références déjà existantes au sein de la base, ou provenant de sources externes, concernant notamment l'histoire du papier (fig. 2). 


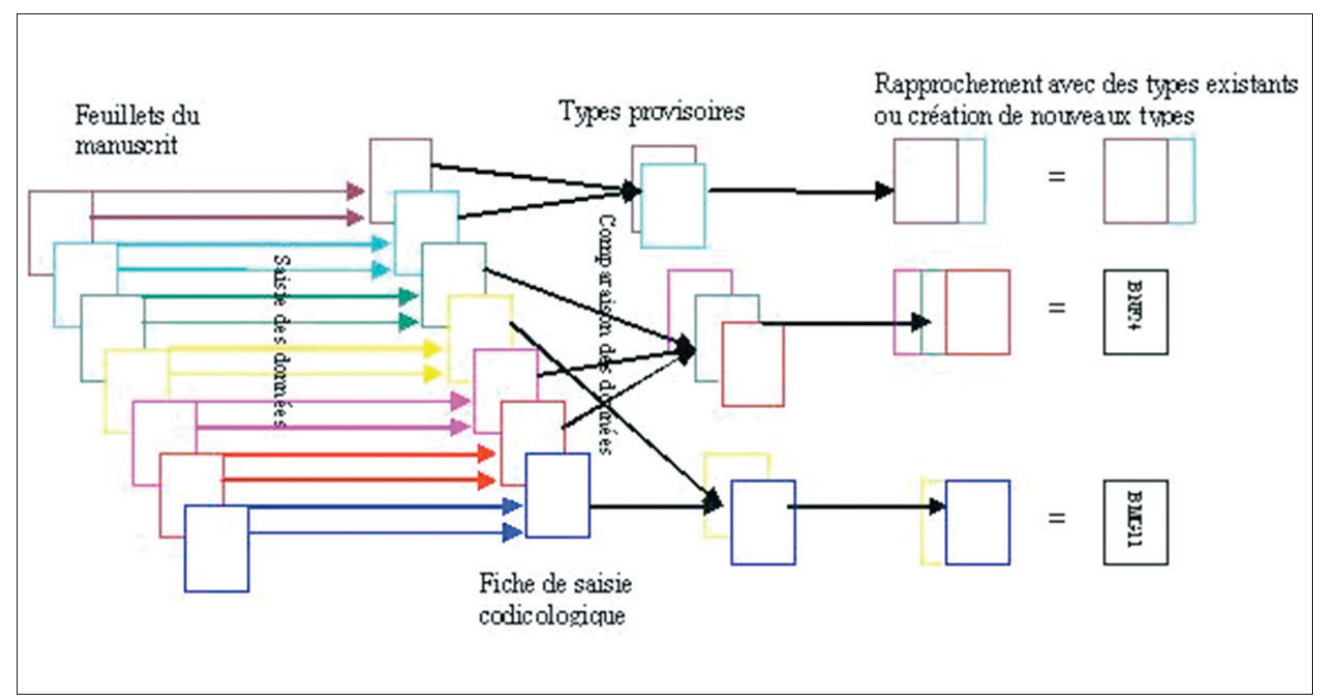

Fig. 2. Processus d'identification des types de papier.

Il faut noter que l'utilisation de la base entraîne d'importantes conséquences dans la méthodologie de la description du manuscrit. Outre une normalisation des critères descriptifs de type qualitatif, le recours à l'outil informatique favorise l'exploitation des données chiffrées (les mesures d'épaisseur et de rugosité donnant lieu à des calculs automatiques de moyennes et d'écarts type). La base de données relationnelle autorise la visualisation comparative d'une quantité d'informations qui dépasse largement nos capacités cognitives, l'accumulation des données rendant la base de plus en plus performante au fur et à mesure du traitement des corpus successifs (consultation des types de papier déjà inventoriés ${ }^{20}$, des informations historiques sur les fabricants, sur les copistes, recoupements avec des occurrences relevées dans d'autres corpus, etc.).

\section{Structure de la base et intégrité référentielle}

Divers fichiers réservés à chaque aspect du manuscrit facilitent la collecte systématique des données concernant les papiers, les filigranes et timbres secs

20. Ainsi lors d'une enquête sur les supports employés par Flaubert et Bouilhet pour la rédaction de Pierrot au sérail, la consultation de la base a permis un recoupement avec certains brouillons de Madame Bovary. Voir M. C. Olds, Au pays des perroquets : féerie théatrale et narration chez Flaubert, Amsterdam, Atlanta, Rodopi, «Faux-titre», 200 I, «Appendice», p. I8 I-I 88 : «Résultats de l'analyse codicologique menée par Cl. Bustarret». 
qui les marquent, les scripteurs et les instruments d'écriture qu'ils emploient, les auteurs et les œuvres traités, les volumes ou autres unités matérielles examinés et les feuillets qui les composent. Enfin, chaque feuillet répertorié comprend nécessairement deux pages, écrites ou vierges, paginées ou non, se prêtant à l'analyse génétique des opérations d'écriture. L'enjeu d'une telle répartition des informations consiste à rationaliser la saisie mais aussi et surtout à favoriser les recoupements entre divers fichiers en réponse à des requêtes multicritères. Le système d'information de MUSE répartit donc dans un fichier précis les données concernant un seul et même type d'entité ('instrument d'écriture, le type de papier, le scripteur...), ce qui autorise la création de relations entre les fichiers et assure l'intégrité référentielle de la base.

Pour assurer la validité des données et des requêtes complexes, MUSE gère l'intégrité référentielle entre les différents fichiers qui la composent ce qui permet d'éviter tout risque de doublons ou d'erreurs d'attribution. Les relations établies entre les fichiers agissent principalement lorsque l'on ajoute ou supprime des enregistrements en éliminant les enregistrements «orphelins». Le respect de ces règles nous garantit donc, par exemple, que chaque folio décrit ne sera associé qu'à un seul type de papier. Ainsi, lors d'une interrogation sur le type de papier ayant pour identifiant «BNF 456 », MUSE fournira uniquement la liste des folios rédigés sur ce type de papier en excluant tous les autres. En effet, tous les fichiers liés entre eux respectant l'intégrité référentielle, il n'y aucun risque que l'on puisse attribuer à un folio plusieurs types de papier (dans le cas d'un folio composite - avec collage(s) par exemple - chaque élément est traité comme un folio unique, sans perdre les informations sur le caractère hétérogène de l'ensemble). Ainsi, les bases de données relationnelles permettent non seulement d'éviter la saisie de données redondantes, mais surtout d'optimiser le recoupement des données lors de requêtes multiples. Parce qu'ils résultent de démarches créatives singulières, et bien souvent de processus peu linéaires, les manuscrits modernes ne se laissent guère appréhender par un cadre prédéfini, transférable d'un corpus à un autre. Aussi le dispositif modulaire et adaptable de la base relationnelle parait-il le mieux adapté pour rendre compte de la complexité propre aux dossiers génétiques.

Nous résumons ci-après deux études de cas qui illustrent concrètement la pertinence de l'examen codicologique et l'efficacité de MUSE dans l'élaboration du dossier génétique : le cas du manuscrit de Lamiel de Stendhal et celui des cahiers d'Impressions d'Afrique de Roussel. 


\section{Le cas Lamiel}

Dans le cas de l'examen du manuscrit de Lamiel $^{21}$, l'ultime roman de Stendhal, l'analyse codicologique des brouillons a produit des résultats inattendus qui ont bouleversé l'ensemble du dossier génétique. En effet, alors que la critique avait établi une chronologie de la rédaction qui n'avait jamais été jusque-là remise en cause, les résultats de l'enquête codicologique ont permis de proposer une datation radicalement différente des diverses campagnes de rédaction. C'est notamment en se fondant sur les recoupements entre la provenance italienne ou française des papiers et les interventions successives de l'auteur, de copistes romains, et du copiste parisien Bonavie - à qui Stendhal avait dicté $L a$ Chartreuse de Parme - que l'on a pu établir une chronologie fort différente des premières phases de la genèse du roman inachevé.

On trouve en effet dans le manuscrit de Lamiel, rédigé dans les derniers moments de la vie de Stendhal, plusieurs sortes de papiers vélins employés de I 839 à I 842 , qui portent un timbre sec identique permettant d'en identifier la provenance commune : «Chambellan, Papetier, I rue du Bouloi à Paris». Dès lors, le recoupement des informations concernant le timbre sec, le papier, le scripteur et le texte nous a permis d'affirmer que Stendhal avait dicté plus de soixante-dix pages de son roman à Paris en mai I 839 au copiste Bonavie, alors que l'on pensait jusqu'ici que ce roman avait été dicté à Civittavecchia à un secrétaire inconnu en octobre I $839^{22}$. C'est bien évidemment le croisement entre l'analyse codicologique complète (supports, encres, instruments d'écriture, et scripteurs) de tous les feuillets concernés, les éléments génétiques relevés par le chercheur, et l'examen critique de l'avant-texte, qui a permis de réorganiser le dossier de genèse de cette œuvre inachevée. Issue de ces découvertes, une nouvelle édition ${ }^{23}$, nous devrions presque dire une nouvelle version du dernier roman de Stendhal, verra le jour prochainement, enrichie par des textes inédits que l'analyse génétique a permis de mettre au jour.

2 I. S. Linkès, «Genèse de Lamiel : le chainnon manquant», thèse de Doctorat sous la direction de B. Didier, Université Paris VIII, 2000. Une partie de cette thèse servira de préface à l'édition du texte chez Champion, voir infra.

22. Sur ce point voir S. Linkès, «Le manuscrit de Lamiel: la fin d'une énigme?», Le dernier Stendhal: $1837-1842$, textes réunis par M. Arrous, Saint-Pierre du Mont, Eurédit, 2000.

23. Stendhal, Lamiel, Romans III, texte établi et annoté par S. Linkès, Gallimard, «Bibliothèque de la Pléiade», à paraittre en 2008. Stendhal, Lamiel, texte établi et annoté par S. Linkès, Champion, «Textes de littérature moderne et contemporaine», collection dirigée par A. Montandon, à paraitre en 2008 (éditions diplomatique papier et électronique). 


\section{Le cas Impressions d'Afrique}

Raymond Roussel rédigea quant à lui les brouillons d'Impressions d'Afrique sur plusieurs cahiers d'écolier d'apparence similaire, qu'il n'a pas conservés dans leur état de reliure initial. Un relevé systématique effectué sur plus de 900 feuillets à l'aide de MUSE décela une dizaine de types de papier différents, et offrit une série d'indices codicologiques indispensables pour reconstituer une genèse mouvementée, que les déclarations publiées par Roussel à titre posthume dans Comment j’ai écrit certains de mes livres n'éclairaient guère. En regroupant virtuellement les occurrences matériellement éparses de chacun de ces types de supports, Anne-Marie Basset cherchait à replacer dans le fil de la genèse de nombreux passages finalement écartés par l'écrivain ${ }^{24}$. Appliquée méthodiquement, la démarche apporta plus de résultats que l'on n'en escomptait, notamment à partir des types de papiers employés par l'écrivain au cours des phases initiales de son travail. Non seulement des séquences suivies jusqu'alors inaperçues furent mises au jour, mais ce faisant l'on découvrait divers aspects spécifiques des habitudes rédactionnelles de Roussel, comme par exemple l'emploi du cahier d'écolier comme d'un bloc, dont il détachait les feuillets au fur et à mesure de leur utilisation. La restitution d'une version continue inédite des Impressions, antérieure au remaniement global des deux parties de l'ouvrage, constituait une véritable découverte en génétique roussellienne, acquise grâce au recours initial à l'examen codicologique.

Ces exemples montrent l'intérêt de la base MUSE sous la forme existante, mais aussi ses limites. En effet, certains aspects de la démarche critique (le déchiffrement et la transcription, le relevé d'indices génétiques tels que ratures, suppressions et déplacements...) n'étaient pas intégrés à la base de données. La nécessité s'impose désormais de rapprocher, au sein d'une même application, l'ensemble des éléments qui permettent d'élaborer un dossier génétique cohérent. Bien souvent, une information codicologique ou génétique demeure inexploitée, voire inexploitable, tant qu'elle ne bénéficie pas de l'enrichissement mutuel provoqué par le rapprochement de ces deux démarches complémentaires. C'est pour systématiser ce rapprochement que nous souhaitons aujourd'hui faire évoluer MUSE en ARGOLIDE.

24. Voir Cl. Bustarret et A.-M. Basset, «Les Cahiers d'Impressions d'Afrique : l'apport de la codicologie à l'étude génétique», Genesis, no 5 , printemps 1994, p. I 53-166, ainsi que A.-M. Basset, «La genèse d'Impressions d'Afrique de R. Roussel ou le mythe de la création», thèse de Doctorat, sous la direction de H. Béhar, Université Paris III, I 996. 


\section{L'avenir de MUSE en ARGOLIDE}

Comme nous l'avons montré, l'approche des manuscrits par la critique génétique requiert un travail important d'observation et de description, préalable au classement des matériaux constituant le dossier de genèse, à la transcription des manuscrits et à l'interprétation des processus d'écriture. Jusqu'à présent ces observations ne donnaient pas lieu à une collecte systématique, ni à une transmission. S'agissant de création littéraire, l'expérience montre pourtant que l'objet intellectuel et l'objet matériel sont indissociables. Ce sont ces deux dimensions, intellectuelle et matérielle, que le projet de transformation de MUSE en ARGOLIDE se propose de concilier afin d'élargir l'assise méthodologique de l'analyse génétique des manuscrits et d'en faciliter la transmission. Malgré les résultats encourageants obtenus avec MUSE, nous sommes conscients que la complexité des relations possibles entre l'analyse des supports et celles des campagnes d'écriture (un même feuillet peut porter plusieurs états génétiques, les brouillons d'une œuvre peuvent être répartis entre plusieurs volumes, une même version peut comporter l'intervention de plusieurs scripteurs sur les mêmes supports ou sur des supports différents, etc.) nécessite la création d'un outil nouveau adapté à la problématique des manuscrits d'auteurs dans son ensemble.

Le projet ARGOLIDE a été élaboré au sein d'un groupe de chercheurs et de doctorants s'accordant sur la nécessité de mettre systématiquement les données matérielles en relation avec les données intellectuelles de l'analyse génétique. À la suite de la présentation des travaux de l'équipe «Techniques et pratiques de l'écrit» lors de l'école thématique «Critique génétique : manuscrits, écriture, invention» du 20 au 24 septembre $2004^{25}$, ce groupe s'est réuni régulièrement à l'ITEM pour réfléchir à la manière la plus efficace de poursuivre les travaux dans ce sens, mettant en commun les expériences sur des corpus fort divers. La participation de l'équipe «Techniques et pratiques de l'écrit» au projet OPTIMA ${ }^{26}$ de l'ITEM, qui intégrera les informations codicologiques à l'enregistrement du document manuscrit sous forme numérique, ne fait que renforcer l'urgence de l'élaboration d'un tel outil. Il est apparu nécessaire, devant ces exigences, de dépasser le stade initial de la base de données codicologique afin de développer une application prenant également en compte la dimension intellectuelle de l'objet manuscrit (texte, éléments

25. ITEM-UMR 8I 32 CNRS-ENS en partenariat avec l'IMEC (Institut Mémoire de l'édition contemporaine).

26. http://www.item.ens.fr, rubrique «Équipes et contrats», «projet OPTIMA». 
génétiques, critiques et graphiques...) et accompagnant la démarche du chercheur dans l'élaboration du dossier de genèse.

C'est pourquoi notre projet actuel vise à combler la triple lacune constatée à l'issue de huit années d'utilisation de la base MUSE, en se donnant un triple objectif :

- compléter et mettre à disposition une base de référence codicologique, manquant à ce jour pour la période moderne et contemporaine (répertoire de produits papetiers, filigranés ou non, et des instruments d'écriture, en particulier pour la période industrielle);

- donner accès à la méthodologie descriptive afin que les chercheurs constituent leurs propres relevés, guidés par une interface intuitive et didactique, se formant ainsi à l'expertise tout en échangeant les résultats de leurs travaux;

- coordonner l'analyse matérielle et l'analyse intellectuelle en vue d'exploiter simultanément les données codicologiques et génétiques pour le classement des brouillons et l'établissement du dossier de genèse.

L'outil en développement prend en considération des fonds manuscrits d'horizons différents, correspondant aux domaines de recherche des membres de l'équipe «Techniques et pratiques de l'écrit» et ceux des équipes de l'ITEM appelées à contribuer au projet dans le cadre d'OPTIMA ${ }^{27}$. Par conséquent, le traitement détaillé s'attachera soit à l'analyse d'un fonds dans son ensemble, soit à une œuvre choisie ou un ensemble cohérent défini par les chercheurs. Cette démarche essentiellement macrogénétique permettra, à terme, de synthétiser les recherches menées sur des corpus d'époques différentes, de confronter les résultats obtenus par différentes équipes constituées autour d'un auteur ou d'un thème particulier. ARGOLIDE constitue une proposition originale au niveau de l'échelle de l'analyse génétique : il s'agit moins de l'étude de variantes, des réécritures locales de segments linguistiques (microgénétique), et pas seulement de l'analyse de phases particulières de la genèse d'une œuvre, mais bien du traitement de grands ensembles, comme l'avait initié MUSE en son temps. Le système d'information d'ARGOLIDE devra en quelque sorte modéliser les compétences, les connaissances et la

27. Les «Rejets» de L'Esprit des lois de Montesquieu et d'autres documents contemporains (BnF et BMU Bordeaux / C. Volpilhac-Auger), les trois volumes des Considérations sur la Révolution de Madame de Staël (BnF/S. Tesser), les brouillons de Lamiel (BM Grenoble/ S. Linkès) et les Journaux et papiers de Stendhal (BM Grenoble / C. Meynard, équipe «Manuscrits de Stendhal»), la correspondance de Denon (Châlon-sur-Saône/E. Williamson), les Écrits de Duchamp (fonds Centre G. Pompidou/Cl. Bustarret), le Précis de Décomposition de Cioran (fonds Doucet/N. Cavaillès), les Trois Contes et l'Éducation sentimentale et cinq carnets connexes de Flaubert (BnF/ équipe Flaubert de l'ITEM), le Cahier 57 et les feuillets connexes de La Recherche de Proust (BnF/ équipe Proust), le dossier de La Jeune Parque, avec feuillets et cahiers connexes de Valéry (BnF/ équipe Valéry). 
méthodologie des experts dans les domaines de l'analyse codicologique (ce à quoi l'élaboration de MUSE a déjà largement contribué), de la description, et du classement génétiques des brouillons.

\section{Un auxiliaire de recherche}

Dans cette perspective plus globale, l'analyse matérielle participera de plein droit à l'examen critique de tous les indices, signes et symboles qui composent le manuscrit. La démarche génétique formalisée par ARGOLIDE consistera à réunir et à confronter toutes les traces matérielles et intellectuelles des processus de la création littéraire afin de les mettre en relation les unes avec les autres, en un faisceau de données structurées, susceptible d'orienter l'interprétation critique. Le manuscrit dans son ensemble, l'objet intellectuel et l'objet matériel, se trouvera donc au début et à la fin de cette chaîne de données, au début comme objet «brut» livré à l'observation, à la fin comme dossier de genèse, objet virtuel structuré, lisible, et disponible pour l'interprétation.

ARGOLIDE sera un outil de recherche conçu comme un système heuristique permettant la gestion d'analyses des données codicologiques et génétiques recueillies et la formulation d'hypothèses. L'utilisation d'un tel outil facilitera les opérations de déchiffrement et de mise en séquence des phases de rédaction par la mise en relation de ce qui est écrit avec les indices matériels propres à chaque feuillet. Les hypothèses produites à l'aide d'ARGOLIDE seront triples :

- codicologiques d'abord, puisqu'elles permettront d'établir une typologie rigoureuse des données matérielles (types de papier, types d'instruments d'écriture...) à partir d'un inventaire méthodique;

- génétiques ensuite, puisqu'elles autoriseront une analyse systématique et affinée des données descriptives portant sur les manuscrits de travail, analyse étayée et confirmée par les résultats de l'enquête codicologique;

- éditoriales enfin, puisqu'elles faciliteront le repérage des strates génétiques de même nature et des différentes versions d'un même texte, le classement chronologique des versions...

Au fur et à mesure de l'enrichissement de la nouvelle base, on peut supposer que le rapprochement de données collectées à partir de manuscrits d'auteurs différents permettra d'enrichir l'étude génétique de chacun d'eux. Ainsi, la reconnaissance de types de papier identiques sous des plumes différentes contribuera à dater des liasses et à reclasser des brouillons : à titre d'exemple plusieurs types de papier rencontrés dans le dossier des Considérations sur la Révolution de Madame de Staël et datés par leur emploi dans d'autres corpus pourront contribuer à situer diverses interventions allographes dans la chronologie. On sait déjà que certains types de papier ont été utilisés à une 
époque contemporaine par différents auteurs : les vélins «Lacroix Frères» se retrouvent vers I 860 chez Zola et Flaubert, des vergés mécaniques «Hachette et $\mathrm{C}^{\mathrm{ie}}$ » ou «Sévigné paper» vers I9Is notamment chez Proust, Roussel et Duchamp. À un stade plus avancé, on peut supposer qu'il sera possible de mener une étude synchronique des processus d'écriture, en comparant par exemple les différents usages du cahier d'écolier dans l'entre-deux-guerres. L'augmentation du nombre de données ne fera que multiplier ces possibilités de recoupement. De même, le fait qu'ARGOLIDE soit conçu pour l'analyse de données codicologiques et génétiques de corpus du XVII au XXe siècle rendra envisageable d'entreprendre le même type d'enquête d'un point de vue diachronique et d'esquisser une histoire de l'évolution des processus d'écriture et des opérations génétiques, histoire dont les brouillons d'écrivains constituent les témoins matériels les plus précieux.

\section{Un outil didactique}

ARGOLIDE se donne aussi pour objectif de combler une lacune qu'ont pu constater les chercheurs s'initiant à l'analyse codicologique et génétique du manuscrit d'auteur. L'absence d'outil informatique dédié contraint souvent ceux-ci à construire leurs propres instruments de travail en utilisant des logiciels de bureautique (au mieux des systèmes de gestion de base de données, parfois des tableurs ou même des traitements de texte) qui n'ont pas été conçus pour l'analyse d'un objet d'étude aussi complexe que le brouillon d'auteur. Outre le temps perdu à tenter d'adapter ces applications propriétaires, ce qui est dommageable pour le chercheur, on s'aperçoit que ces outils, construits en fonction d'un corpus donné et d'une problématique spécifique, peuvent rarement être transmis à d'autres chercheurs, ce qui est dommageable pour la discipline elle-même.

L'intérêt d'ARGOLIDE sera donc d'offrir un auxiliaire de recherche simple et adaptable à des objets d'étude tous différents par nature et de permettre notamment aux doctorants de maitriser plus rapidement leur corpus afin de se concentrer sur l'analyse critique et non sur l'élaboration d'outils. Plus précisément, cet auxiliaire offrira non seulement une méthodologie en proposant une démarche structurée, il fournira aussi la possibilité de se référer aux données déjà engrangées sur d'autres corpus. 


\section{Un outil d'aide à la diffusion}

Le projet OPTIMA de l'ITEM sélectionné par l'ANR souhaite répondre à l'intérêt grandissant que porte le public à l'archive littéraire en s'attelant au projet d'une vaste édition numérique des manuscrits littéraires. De ce point de vue, les ambitions d'ARGOLIDE sont plus modestes : cet outil doit seulement faciliter la démarche éditoriale en permettant l'exportation des données (texte, image, appareil critique...) pour réaliser des éditions papier ou numériques, voire des bases de connaissances consultables sur Internet.

Cependant la mise en ligne, sur laquelle nous nous interrogeons actuellement, est complexe puisqu'elle nécessite un travail de présentation des documents manuscrits dans leur dimension matérielle et intellectuelle et l'établissement d'un paratexte critique standardisé dans la forme - par la normalisation inévitable de la mise en ligne - mais particulier à chaque manuscrit dans le fond - du fait de l'hétérogénéité intrinsèque de l'objet. Alors que la numérisation et la mise en ligne tendent finalement à «dématérialiser» l'objet manuscrit, transformé en image, l'originalité du projet ARGOLIDE consiste bien à rendre accessible, par le biais d'une description détaillée, la dimension matérielle de l'original et à en exploiter la teneur informative, trop souvent négligée par les projets de numérisation entrepris à grande échelle. 